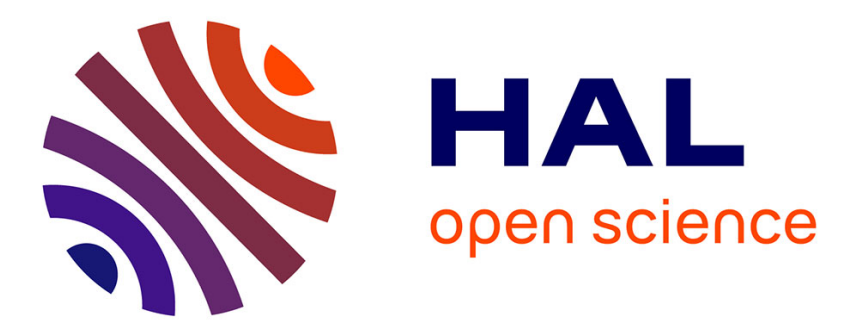

\title{
Equilibrium and Out-of-Equilibrium Adherence of Hydrogels against Polymer Brushes
}

Jennifer Macron, Bruno Bresson, Yvette Tran, Dominique Hourdet, Costantino Creton

\section{To cite this version:}

Jennifer Macron, Bruno Bresson, Yvette Tran, Dominique Hourdet, Costantino Creton. Equilibrium and Out-of-Equilibrium Adherence of Hydrogels against Polymer Brushes. Macromolecules, 2018, 51 (19), pp.7556 - 7566. 10.1021/acs.macromol.8b01063 . hal-01904369

\section{HAL Id: hal-01904369 \\ https://hal.science/hal-01904369}

Submitted on 23 Dec 2018

HAL is a multi-disciplinary open access archive for the deposit and dissemination of scientific research documents, whether they are published or not. The documents may come from teaching and research institutions in France or abroad, or from public or private research centers.
L'archive ouverte pluridisciplinaire HAL, est destinée au dépôt et à la diffusion de documents scientifiques de niveau recherche, publiés ou non, émanant des établissements d'enseignement et de recherche français ou étrangers, des laboratoires publics ou privés. 
This document is confidential and is proprietary to the American Chemical Society and its authors. Do not copy or disclose without written permission. If you have received this item in error, notify the sender and delete all copies.

\section{Equilibrium and out-of-equilibrium Adherence of Hydrogels against Polymer Brushes}

\begin{tabular}{|r|l|}
\hline Journal: & Macromolecules \\
\hline Manuscript ID & Draft \\
\hline Manuscript Type: & Article \\
\hline Date Submitted by the Author: & n/a \\
\hline Complete List of Authors: & $\begin{array}{l}\text { Macron, Jennifer; ESPCI Paris, SIMM } \\
\text { Bresson, Bruno; ESPCI, CNRS, LPQ/ESPCI } \\
\text { Tran, Yvette; ESPCI Paris, SIMM } \\
\text { Hourdet, Dominique; SIMM, Université Pierre et Marie Curie, Sorbonne- } \\
\text { Universités, } \\
\text { Creton, Costantino; E.S.P.C.I., Laboratoire SIMM-UMR 7615 }\end{array}$ \\
\hline
\end{tabular}

SCHOLARONE ${ }^{m}$

Manuscripts 


\title{
Equilibrium and out-of-equilibrium Adherence of Hydrogels against Polymer Brushes
}

\author{
Jennifer Macron ${ }^{\mathrm{a}, \mathrm{c}}$, Bruno Bresson ${ }^{\mathrm{a}}$, Yvette Tran ${ }^{\mathrm{a}}$, Dominique Hourdet ${ }^{\mathrm{a}, \mathrm{b}}$ and Costantino \\ Creton $^{\mathrm{a}, \mathrm{b}, *}$
}

\author{
${ }^{a}$ Laboratoire Sciences et Ingénierie de la Matière Molle, ESPCI Paris, PSL University, \\ Sorbonne Université, CNRS, F-75005 Paris, France \\ ${ }^{\mathrm{b}}$ Global Station for Soft Matter, Global Institution for Collaborative Research and Education, \\ Hokkaido University, Sapporo, Japan \\ ${ }^{c}$ current address: Bertarelli Foundation Chair in Neuroprosthetic Technology, Laboratory for Soft \\ Bioelectronic Interfaces, Institute of Microengineering, Institute of Bioengineering, Center for \\ Neuroprosthetics, Ecole Polytechnique Fédérale de Lausanne (EPFL), 1202 Geneva, Switzerland \\ emails: Jennifer.Macron@epfl.ch, Bruno.Bresson@espci.fr, Yvette.Tran@espci.fr, \\ Dominique.Hourdet@espci.fr, Costantino.Creton@espci.fr
}




\begin{abstract}
$\underline{\text { ABSTRACT }}$
At low $\mathrm{pH}$, underwater adherence between poly $(N, N$-dimethylacrylamide) hydrogels and poly(acrylic acid) brushes is due to the formation of multiple hydrogen bonds. The effect of the key parameters controlling the formation of these interactions (contact time and composition of the hydrogel) was investigated with a contact mechanics test using a flat probe. We specifically quantified the loss of adherence during the progressive swelling to equilibrium of the gels and, at fixed contact time, found a significant decrease in adherence between the preparation state and the swelling equilibrium even in the case of relatively low dilution factors. This adherence loss was attributed to the slowdown of the kinetics of formation of multiple H-bond interactions as the gel approached its equilibrated state. In both limiting conditions the energy of adherence scaled with the polymer concentration, independent of the crosslinks density of the gel, suggesting that the Lake and Thomas amplification factor is not relevant for these weak bonds.
\end{abstract}

Keywords: underwater adherence, hydrogels, polymer brush, weak interactions, out-of-equilibrium, swelling equilibrium.

\title{
$\underline{\text { TOC }}$
}
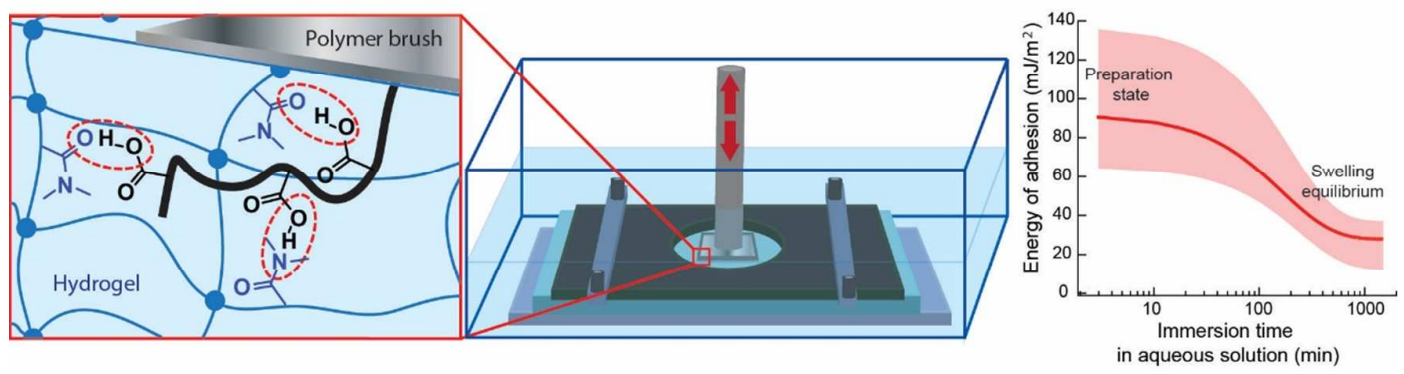


\section{INTRODUCTION}

The use of hydrogels as surgical adhesives ${ }^{1}$, drug-loaded skin or mucoadhesives ${ }^{2}$, injectable adhesive hydrogels ${ }^{3}$ or tailor-made artificial organs through $3 \mathrm{D}$ bioprinting ${ }^{4}$ has raised some fundamental questions about the hydrogel adherence with mucus in wet environments and its evolution over time. More recently some work has been done to control and improve the adherence of synthetic hydrogels to hydrophilic and hydrophobic surfaces for the engineering purpose of assembling multimaterials ${ }^{5-8}$. However, in all these cases, the main objective was to design and demonstrate a recipe or method that works, rather than to obtain a deep and general understanding of the underlying principles behind gel adherence.

In parallel some more systematic studies have also been carried out with model systems ${ }^{9-11}$, investigating among other things the effect of the detailed composition of the gel, of the environment ( $\mathrm{pH}$ in particular), ionic strength or temperature on the measured adherence with the objective to develop robust models. Our study fits well in this latter category and focuses on the process of equilibration of the gel with its environment and its consequences on adhesive interactions.

Synthetic hydrogels are model hydrophilic soft materials that are typically swollen with water (80$95 \%$ of water) and have moduli in the $\mathrm{kPa}$ range. They are normally composed of flexible watersoluble polymer chains synthesized or crosslinked in the presence of a fixed concentration of water (constituting the preparation conditions) and, if left then in excess water, will swell to equilibrium. This equilibration with the environment until the chemical potential of the water and mobile species inside and outside the gel is the same, has been well studied and leads to changes in mechanical properties such as the elastic modulus and also to changes in the large strain properties ${ }^{12-13}$. However changes in surface properties and adherence due to this equilibration process in aqueous conditions have not been addressed systematically to the best of our knowledge.

The process of swelling to equilibrium of the polymer gel results in a dilution of the flexible chains and therefore in a reduction in their concentration. In the absence of heterogeneities, this decrease in polymer concentration also occurs on the surface of the hydrogel.

Taking a naïve picture of adherence, one can hypothesize that if adherence between two hydrogels or between a hydrogel and a polymer brush occurs at preparation conditions and at swelling equilibrium, the areal density of interactions should scale with the polymer concentration. When the hydrogel is separated from the surface in controlled conditions, the energy needed to perform this debonding test will in turn depend on the strength and areal density of these interactions (the stress that can be sustained by the interface) and also on the bulk dissipative mechanisms in the hydrogel. If the interactions are weak and the gels are elastic, bulk dissipation is negligible. In this case, the energy dissipated during the debonding (which we call work of adherence) can be attributed to the irreversible energy dissipation occurring during the breakup of weak bonds attached to flexible chains. This scenario has been discussed in detail by Chaudhury for hydrophobic systems ${ }^{14-15}$ and assumes that this 
bond scission is rate dependent as proposed originally by Evan Evans ${ }^{16}$, and depends also on the length of the chain to which the interacting group is attached, as proposed by Lake and Thomas ${ }^{17}$ for the scission of main chain bonds in crosslinked flexible networks.

It is the purpose of this article to explore different conditions under which underwater macroscopic adherence can be measured between a neutral hydrogel and a polymer brush in conditions where gel/brush molecular interactions can occur by hydrogen bonding. The model gel is made of poly $(N, N-$ dimethylacrylamide) (PDMA) and the brush is made of poly(acrylic acid) (PAA). As reported previously this type of combination gives a weak and reversible adherence which depends strongly on the $\mathrm{pH}$ and temperature of the medium ${ }^{11,18}$. Above the $\mathrm{pK}_{\mathrm{a}}$ of the acid/base functions of the brush, the carboxylic groups of PAA are negatively charged and no adherence is observed with neutral PDMA. However in acidic conditions (well below the $\mathrm{pK}_{\mathrm{a}}$ of the acid/base functions), the PAA brush is fully protonated and multiple hydrogen bonds can be formed between the brush and the gel, even in water, leading to a measurable macroscopic adherence.

In a previous study ${ }^{11}$, we investigated the dependence of $\mathrm{pH}$ on the underwater adherence of neutral hydrogels swollen at equilibrium, i.e. in a situation where brush and gel were immersed for several hours in the medium before being put in contact. In those conditions, the adherence was measured below $100 \mathrm{~mJ} / \mathrm{m}^{2}$ and required several minutes of contact to be observed.

We address here more specifically two important aspects: the variation in adherence energy as the gel preparation conditions are varied (concentration of monomer and degree of crosslinking) and the variations in the observed adherence energy as the gel, initially in preparation conditions, progressively swells to equilibrium in an acid solution.

\section{EXPERIMENTAL SECTION}

\section{$\underline{2.1 \text { Mold design for hydrogel layer preparation }}$}

Performing a flat-flat adherence test between a hydrogel and a nanometrically smooth surface such as a polymer brush requires a macroscopic gel with a low amplitude surface roughness. To obtain this low roughness, we used a mold formed by two hydrophobically modified glass substrates (dimensions: $75 \times 25 \mathrm{~mm}$ ), separated by a silicone rubber gasket (1.8 mm thick) and held together using binder clips (see suppl. info Figure S1). The hydrophobic substrates were obtained by chemically grafting octadecyltrichlorosilane (OTS, Sigma-Aldrich, $\geq 90 \%$ ) on glass. Before any chemical modification, the glass substrates were cleaned in a "Piranha" solution (mixture of 70 vol \% of sulfuric acid (SigmaAldrich, 97\%) and 30 vol\% of hydrogen peroxide (Sigma-Aldrich, $35 \%$ )), heated at $150{ }^{\circ} \mathrm{C}$ for 30 minutes, then rinsed, sonicated in Milli-Q water bath and dried under nitrogen flow. Silanization occurred using a liquid phase method: clean substrates were immersed in a sealed reactor for 2 hours 
at room temperature in a $0.5 \mathrm{vol} \%$ OTS solution prepared in extra-dry toluene. The glass substrates were then removed from the bath, rinsed with Milli-Q water and finally dried under nitrogen flow.

\subsection{Synthesis of the PDMA hydrogels}

The synthesis of poly( $N, N$-dimethylacrylamide) hydrogels was carried out in Milli-Q water at room temperature by free radical polymerization of $x \mathrm{wt} \%$ of $N, N$-dimethylacrylamide (DMA, SigmaAldrich, 99\%) monomers with $y$ mol\% of N,N'-methylenebisacrylamide (MBA, Sigma-Aldrich, 99\%) as cross-linkers. As redox initiator system, we used $1 \mathrm{~mol} \%$ potassium persulfate (KPS, SigmaAldrich, $\geq 99 \%$ ) and 1 mol\% N,N,N',N'-tetramethylenediamine (TEMED, Sigma-Aldrich, 99\%). After the dissolution of monomer, cross-linker and KPS in Milli-Q water, the solution was deoxygenated for 30 minutes with a bubbling of nitrogen. TEMED was then rapidly added to the formulation under stirring, and the solution was directly poured into the mold described in the previous section that was placed in a reactor under a nitrogen atmosphere, to avoid oxygen inhibition of radicals. Polymerization and cross-linking occurred simultaneously, and molds were left in the reactor for 10 hours at room temperature to ensure complete reaction. The hydrogels were kept into the mold closed with paraffin film for a maximum of 3 days, to avoid water evaporation, before being used for swelling and adhesive tests. The resulting synthesized gels will be referred to as: Px-CLy, with $x$ being the polymer concentration in wt $\%$ and $y$ the cross-linking ratio in mol\% relative to monomer. Table 1 summarizes the composition of the $\mathbf{P} x$-CL $y$ hydrogels that were synthetized for this study.

\begin{tabular}{ccccccc}
\hline $\begin{array}{c}\text { P } x \text {-CL } y \\
\text { PDMA hydrogels }\end{array}$ & DMA/g & $\mathrm{H}_{2} \mathrm{O} / \mathrm{g}$ & $\mathrm{KPS} / \mathrm{mg}$ & $\mathrm{MBA} / \mathrm{mg}$ & $\mathrm{TEMED} / \mu \mathrm{L}$ & $\begin{array}{c}\text { Initial mass } \\
\text { swelling } \\
\text { ratio } \Lambda_{0}\end{array}$ \\
\hline P10-CL1 & 1 & 9 & 27.3 & 15.6 & 15.2 & 10.3 \\
P10-CL2 & 1 & 9 & 27.3 & 31.1 & 15.2 & 10.2 \\
P10-CL3 & 1 & 9 & 27.3 & 46.7 & 15.2 & 10.0 \\
P15-CL3 & 1.5 & 8,5 & 40.9 & 70.0 & 22.8 & 6.6 \\
P20-CL3 & 2 & 8 & 54.5 & 93.3 & 30.4 & 4.9 \\
P30-CL3 & 3 & 7 & 81.8 & 140 & 45.7 & 3.3 \\
\hline
\end{tabular}

Table 1: Chemical composition of P $x$-CLy PDMA hydrogels and swelling ratio in their preparation state.

\section{$\underline{2.3 \text { Kinetics and Swelling Measurements }}$}

The swelling behavior of the PDMA hydrogels was studied from the preparation state to swelling equilibrium as a function of immersion time. To quantify the kinetics of swelling, layers of PDMA hydrogels (dimensions: $73 \times 23 \times 1.8 \mathrm{~mm}^{3}$ ) were weighed in their preparation state before immersion 
into a large volume of acid solution at $\mathrm{pH} 2$ (adjusted by adding hydrochloric acid). The sample was regularly weighed until reaching its swelling equilibrium. After the study of the swelling kinetics, the sample was dried for 48 hours in a desiccator under vacuum at $80^{\circ} \mathrm{C}$ and the dry weight of polymer was measured. The mass swelling ratio $\Lambda(t)$ as a function of immersion time was calculated from the weight data obtained with Equation 1. The results of these bulk swelling experiments are displayed in Figure S2 of the supplementary information.

$$
\Lambda(t)=\frac{m_{\text {gel }}(t)}{m_{\text {polymer }}^{\text {dry }}}=\frac{m_{\text {polymer }}^{0}+m_{\text {water }}(t)}{m_{\text {polymer }}^{\text {dry }}}
$$

\subsection{Synthesis of PAA brushes}

The synthesis of PAA brushes on silicon wafers using the "grafting onto" approach has been described in more details in a previous paper ${ }^{19}$. Briefly, a self-assembled monolayer of 3glycidoxypropyltrimethoxysilane (GPS) was first formed. In a second step, the grafting of poly(tertbutyl acrylate) (PtBA) chains was obtained after the esterification of PtBA-COOH with surfaceattached GPS. In the final step, the PtBA brushes were converted into PAA brushes by a pyrolysis reaction. The PAA brushes we prepared for our study of adherence as a function of immersion time, were $6 \mathrm{~nm}$-thick in the dry state with a grafting density of 0.15 chains/ $\mathrm{nm}^{2}$ and a degree of polymerization DP 300 .

\subsection{Mechanical Characterization}

Uniaxial compression tests were performed on PDMA hydrogel cylinders with an Instron model 3340 fitted with a $10 \mathrm{~N}$ load cell in both preparation and equilibrated states. The preparation state gels were $10 \mathrm{~mm}$-high cylinders with an $8 \mathrm{~mm}$-diameter. The samples were coated with paraffin oil both to avoid water evaporation and to lubricate the interface between the gel and the testing plates during the uniaxial compression. The compression tests were performed at a constant displacement rate of 50 $\mu \mathrm{m} . \mathrm{s}^{-1}$ corresponding to $\dot{\lambda}=0.05 \mathrm{~s}^{-1}$. Swollen samples were tested after a week of swelling in a $\mathrm{pH} 2$ aqueous solutions (the $\mathrm{pH}$ was adjusted using hydrochloric acid) that were changed every day. Dimensions of the swollen gel samples during testing depend on their swelling ratio; for each swollen cylinder, the height and diameter were measured with a caliper before testing and used for the data treatment.

\subsection{Characterization of hydrogel free surfaces by AFM}

Atomic Force Microscopy measurements in water (at $25^{\circ} \mathrm{C}$ ) were performed on a ICON Microscope equipped with a Nanoscope V controller (Bruker). Both out-of-equilibrium and swollen P20-CL3 gels 
were investigated. The probe used for the measurement was a pyramidal silicon nitride nanometric tip of $20 \mathrm{~nm}$ radius and $6 \mu \mathrm{m}$ height. The QNM (Quantitative NanoMechanics) was used for both conditions (out-of-equilibrium and swollen states) with a cantilever stiffness of $0.1 \mathrm{~N} / \mathrm{m}$ (Figure 1). The setpoint force was $2 \mathrm{nN}$, the scanning frequency was $1 \mathrm{~Hz}$ and the oscillation frequency of the cantilever was $1 \mathrm{kHz}$. The gel was held by a Teflon coated plate with a centered circular hole, preventing sliding of the gel during the acquisition. For equilibrium conditions, the swollen hydrogel was supported on a glass substrate and surrounded by a pH 2 acid solution in a Petri dish (Figure 1b). In the preparation state, the gel was surrounded by paraffin oil to avoid water evaporation (Figure 1a). In both cases, the contact between the cantilever and the gel surface was established thanks to a Milli$\mathrm{Q}$ water droplet deposited at the interface.

A (i) Water droplet

(ii)
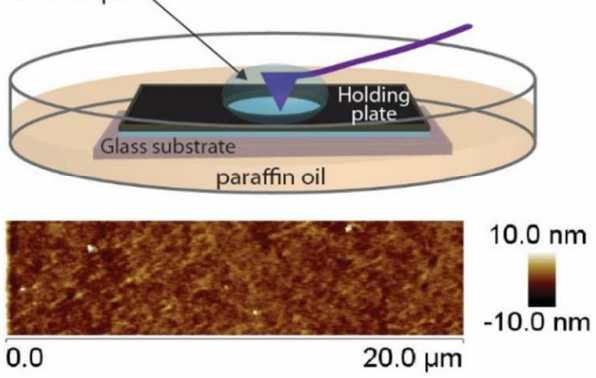

(iii)

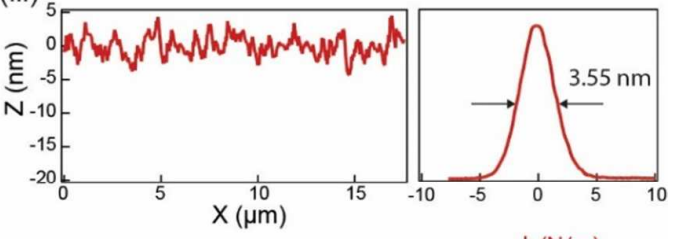

B (i)

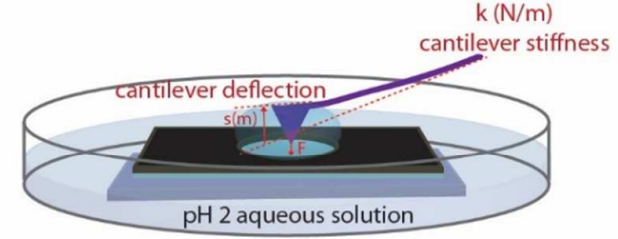

(ii)

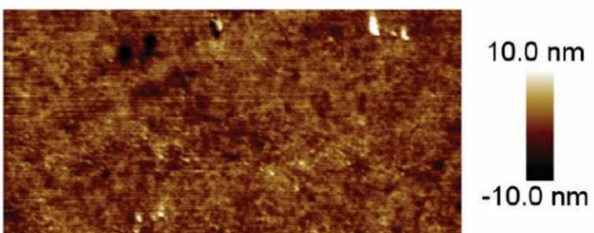

(iii)

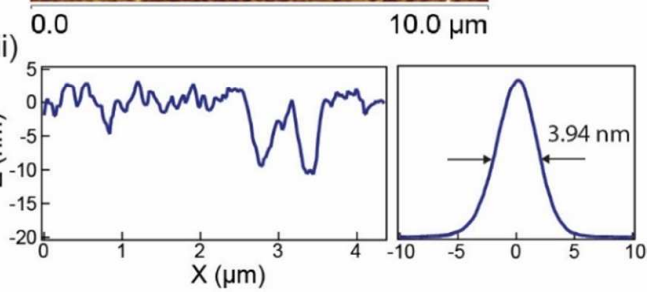

Figure 1:

Characterization of the free surface of the gel by Atomic Force Microscopy (AFM). The AFM experiments were performed on a P20-CL3 hydrogel in water using a cantilever stiffness of $0.1 \mathrm{~N} / \mathrm{m}$. The schemes of the setup are illustrated for the preparation state (A) and in $\mathrm{pH} 2$ aqueous solution (B). Also are displayed the AFM images in QNM modes (ii) and the corresponding height profile and histogram with the mean roughness (iii). 


\subsection{Adherence testing}

To perform the adherence tests we have used an experimental setup based on the flat-flat contact test and specifically designed by Sudre et $a l .^{11}$ to characterize the underwater adherence of soft materials. The full description of this device has been detailed in a previous paper, and we only recall here the main elements necessary for a proper understanding of our present work. The general principle is the same as that of a regular probe test, with some modifications to address the needs of working underwater with swollen hydrogels. Hence, the setup was designed to be able to work both in air (for alignment purposes) and in immersed conditions. The temperature and $\mathrm{pH}$ of the liquid medium are easily adjustable in situ and measurable without disturbing the test. A commercial Instron machine model 3340 was used for all the adherence tests. The load cell of $10 \mathrm{~N}$ is sensitive enough to measure very small forces reproducibly. The noise level of the load cell is on the order of $0.1 \mathrm{mN}$.

To conduct the adherence test, we used a planar silicon wafer of $1 \times 1 \mathrm{~cm}^{2}$ glued on the flat part of a stainless steel cylindrical punch with a polystyrene solution (45 wt $\%$ in toluene) and functionalized on its smooth front side with a PAA brush. The punch was fixed to the load cell with a mandrel and could be pulled off at a constant velocity. During the experiment, the displacement of the punch and the force applied on it are measured as a function of time.

To perform measurements on non-equilibrated hydrogels during swelling, we developed a new sample-holder system, efficiently maintaining the sample from the preparation state to its swelling equilibrium (Figure 2A). A layer of soft hydrogel is laid on a glass substrate and mechanically held by a Teflon-coated aluminum plate, presenting a circular opening of $18 \mathrm{~mm}$ of diameter and clamped with Plexiglas jaws in the trough of the initial setup (Figure 2). As the diameter of the hole is large enough, the mechanical holding has no significant consequences on the swelling of the maintained gel compared to the swelling of a free sample. The modified hard substrate attached on the mobile punch is moved down to come into contact with the soft material and subsequently removed at a constant velocity. 


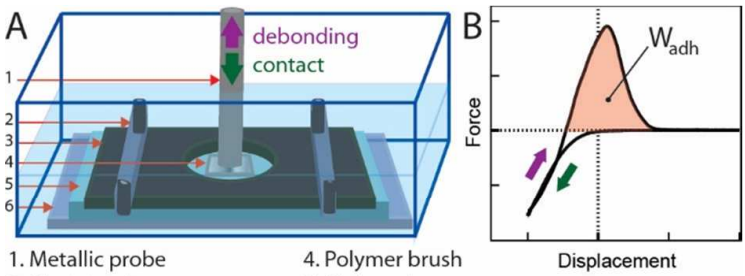

2. Plexiglass jaws $\quad 5$. Hydrogel

3. Teflonated holding plate 6 Supporting glass plate
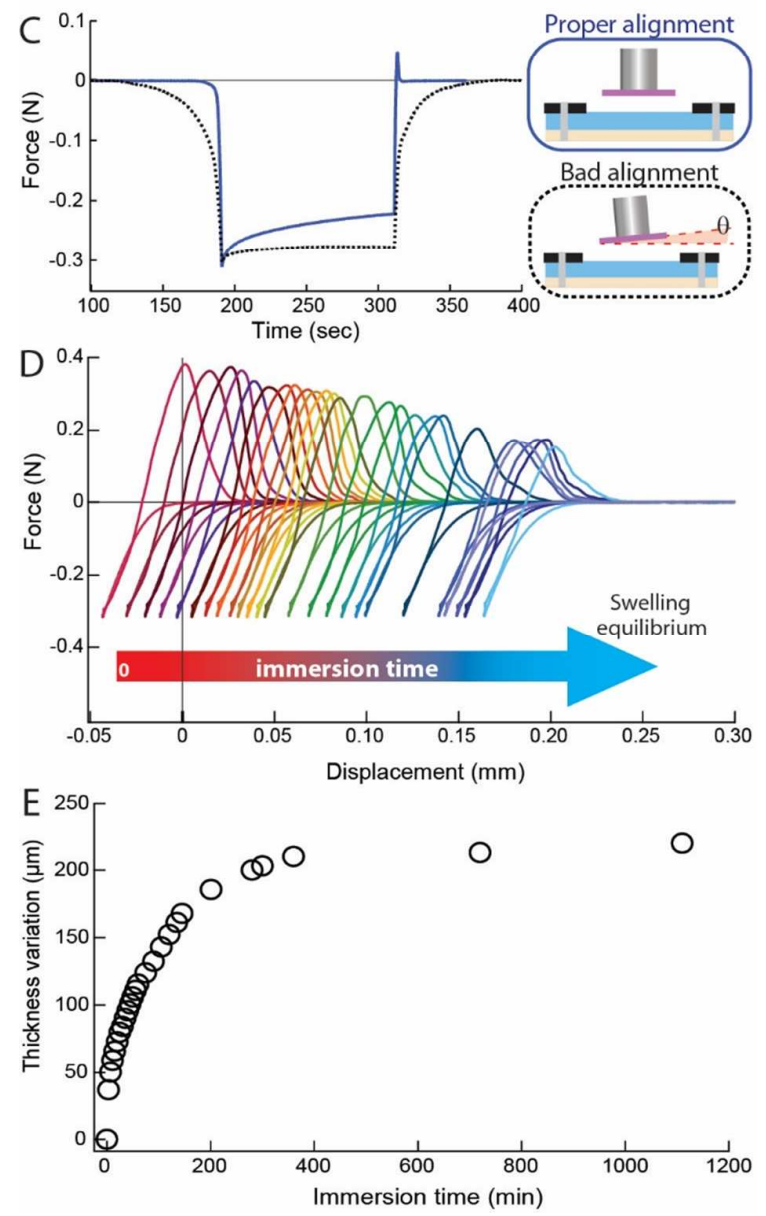

Figure 2: Underwater adherence test between PDMA hydrogel and PAA brush. (A) Illustration of the setup used to mechanically hold the hydrogel layer with a Teflon-coated plate during the adherence test. (B) Typical force-displacement curves obtained during an adherence test and used to calculate the energy of adherence $\mathrm{W}_{\text {adh }}$ (red area). (C) Consequences of the alignment between the hydrogel and the polymer brush on the force response in aqueous solution during the contact and the detachment steps for a correct alignment $(-)$ and for a

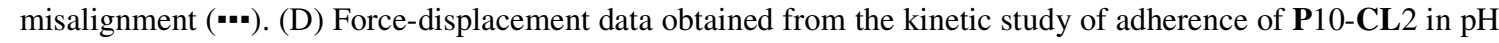
2 aqueous conditions at $\mathrm{T}=21^{\circ} \mathrm{C}$ from the preparation state to the swelling equilibrium of the hydrogel. Each force-displacement curve corresponds to a different immersion time point of the gel. (E) Increase of thickness of P10-CL2 hydrogel during the adherence test in $\mathrm{pH} 2$ aqueous conditions at $\mathrm{T}=21^{\circ} \mathrm{C}$ as a function of the immersion time.

The reproducibility and therefore the quantitative aspect of the adhesive measurements are dependent on the quality of the alignment between the hydrogel and the wafer surface. This alignment has to be done in air (to see the contact) and can be finely tuned with the help of three micrometric screws placed under the device. The punch was approached to the gel surface and the contact was observed for a contact force lower than the values used for the adherence tests. If properly aligned, the two 
materials come into contact, with a contact area equal to the area of the silicon wafer. In that case, the molecular adhesion at the interface causes a peak of force that appears during the detachment step. In the case of misalignment, where the angle between the two surfaces $\theta$ is significant, we observed a lack of adhesive response of the system during the detachment of the punch; the parts of the punch taking off that could contribute to the global adhesive force are offset by those still under compression. An alternative way to check the alignment is the time required to reach a set compressive force. When punch and gel are properly aligned, the time is at a minimum (less than 4 seconds for an approach speed of $10 \mu \mathrm{m} / \mathrm{s}$ in our case). We considered that the alignment was adequate when the contact was complete for a stress lower than $20 \%$ of the contact stress applied during the actual adherence test (see Figure 2C).

After adjusting the alignment, the setup was immersed in an aqueous solution at $\mathrm{pH} 2\left(\right.$ at $21^{\circ} \mathrm{C}$ ) and the immersion time was monitored. After 3 minutes of immersion $\left(t_{i m m}^{1}\right)$, a first measurement at the preset experimental conditions (e.g. with a compressive contact stress of $0.3 \mathrm{~N}$ and a contact time of 2 minutes fixed for all the adhesive tests unless explicitly mentioned) was carried out. This measurement corresponds to the minimum dilution of the PDMA hydrogel immersed underwater (i.e. initial out-ofequilibrium state). The speed at which the probe moves during the compression and detachment stage has been set respectively at $10 \mu \mathrm{m} . \mathrm{s}^{-1}$ and $100 \mu \mathrm{m} . \mathrm{s}^{-1}$ for all the cycles of contact-detachment that have been repeated regularly during 16 hours (see Figure 2D) as the gel sample swells and increases in thickness (Figure 2E). The last measurement of adherence has been performed when the PDMA hydrogel has reached its swelling equilibrium.

Following the convention used for soft adhesives, the adherence energy is obtained from the raw data according to Equation 2 and the scheme in Figure 2B.

$$
W_{a d h}=\frac{1}{A} \int_{x>x_{0}} F(x) \cdot d x
$$

where $\mathrm{A}$ is the contact area between the brush and the hydrogel and $\mathrm{F}$ is the force needed to apply to completely detach the brush from the surface of the PDMA gel.

\section{RESULTS}

\subsection{Effect of contact time on adherence}

Before we report the changes in adherence as the gel swells, we need to address the question of the effect of contact time on adherence in equilibrium conditions. For this purpose, adherence tests were carried out between a fully equilibrated P10-CL2 gel and a PAA brush for different contact times (from 10 seconds to 17 hours). The results of these tests are presented on Figure 3 and show a progressive increase of $\mathrm{W}_{\text {adh }}$ with contact time. Given the thickness of the swollen polymer brush $(\sim 12$ 
$\mathrm{nm}$ ), the time needed for the complete diffusion of the grafted chains inside the hydrogel do not exceed a few milliseconds and cannot be responsible for such a slow dependence of $\mathrm{W}_{\text {adh }}$ with contact time.

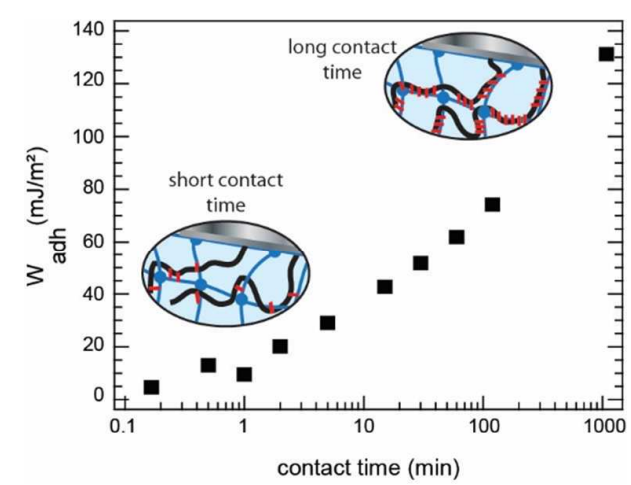

Figure 3 : Energy of adherence as a function of the contact time between the PAA polymer brush and the surface of a P10-CL2 PDMA hydrogel swollen at equilibrium in an aqueous solution at $\mathrm{pH} 2$.

In this system, the energy of adherence between the two surfaces is due to the formation of hydrogen bonds between the PAA brush and the PDMA hydrogel. At short contact times, the measured energy of adherence is quite small $\left(\mathrm{W}_{\mathrm{adh}} \sim 0\right.$ for $\left.\mathrm{t}_{\mathrm{c}}=10 \mathrm{sec}\right)$. This suggests that while individual hydrogen bonds may form quite rapidly, they also have a very short lifetime (few picoseconds). Increasing the contact time enhances the probability to create multiple hydrogen bonds that now have an increasingly longer lifetime and are increasingly stable. In turn, the creation of multiple hydrogen bonds limits the mobility of the polymer in the brush and in the hydrogel so that further increase in multiple bonds slows down logarithmically. The energy of adherence of the equilibrated system is only sensitive to the more stable bonds and increases continuously with contact time to reach, after 17 hours of contact, a level of adherence $\mathrm{W}_{\mathrm{adh}} \sim 130 \mathrm{~mJ} \cdot \mathrm{m}^{-2}$. For the swelling equilibrium we needed to use a contact time long enough to measure some adherence but significantly shorter than the equilibrium swelling time and we picked 120 seconds.

\subsection{Effect of immersion time on the adherence between hydrogel and brush}

Now that the effect of contact time has been addressed, the evolution of the energy of adherence between a polymer brush and a hydrogel was determined during its progressive swelling from its preparation conditions to equilibrium into water. To check the methodology, we first performed the test with a $1.8 \mathrm{~mm}$ thick P10-CL2 hydrogel layer in its preparation state in contact with a $12 \mathrm{~nm}$ thick swollen PAA brush. The physical adhesion at the interface of the brush and the hydrogel is provided at pH 2 by the formation of multiple hydrogen bonds between the chains of PDMA and PAA, through their hydrophilic sites ${ }^{11}$. 
To measure the evolution of the energy of adherence of the out-of-equilibrium system, cycles of compression/traction between P10-CL2 gel and the PAA brush have been repeated every 3 minutes during the first hour of immersion and then every $30 \mathrm{~min} / 1 \mathrm{hr}$ during the next sixteen hours, applying the test parameters defined in the experimental section, i.e. a compression force of $0.3 \mathrm{~N}$, contact time of 120 seconds and a debonding velocity of $100 \mu \mathrm{m} / \mathrm{s}$. The origin of the displacement for the set of experiments has been defined as the contact point between the two surfaces at zero-force before immersion. The raw data obtained from this study, representing the force applied on the gel as a function of the displacement of the probe (Figure 2D), have been used to calculate the energy of adherence of the system $\left(W_{a d h}\right)$ for each cycle as a function of immersion time $\left(t_{i m m}\right)$ (Figure 4$)$. The evolution of the position of the first contact point at zero-force between the two surfaces during the test series is directly related to the increase in thickness of the P10-CL2 gel as it progressively swells $\left(\mathrm{T}=21^{\circ} \mathrm{C}, \mathrm{pH}\right.$ 2) (Figure $2 \mathrm{E}$ ). The change in thickness of the gel has been used to estimate the in situ degree of swelling of the P10-CL2 hydrogel during the adhesive test. In this experiment performed for 5 different P10-CL2/PAA systems, the average energy of adherence continuously decreased from 90 mJ.m $\mathrm{m}^{-2}$, estimated after $3 \mathrm{~min}$ of immersion in the aqueous medium (time referred as $t_{\text {imm }}^{1}$ ), to an average minimal value of $28 \mathrm{~mJ} . \mathrm{m}^{-2}$ after 16 hours of immersion. At this point, the gel is swollen to equilibrium and the energy of adherence measured between P10-CL2 and the PAA brush, for those contact and debonding conditions remained constant.

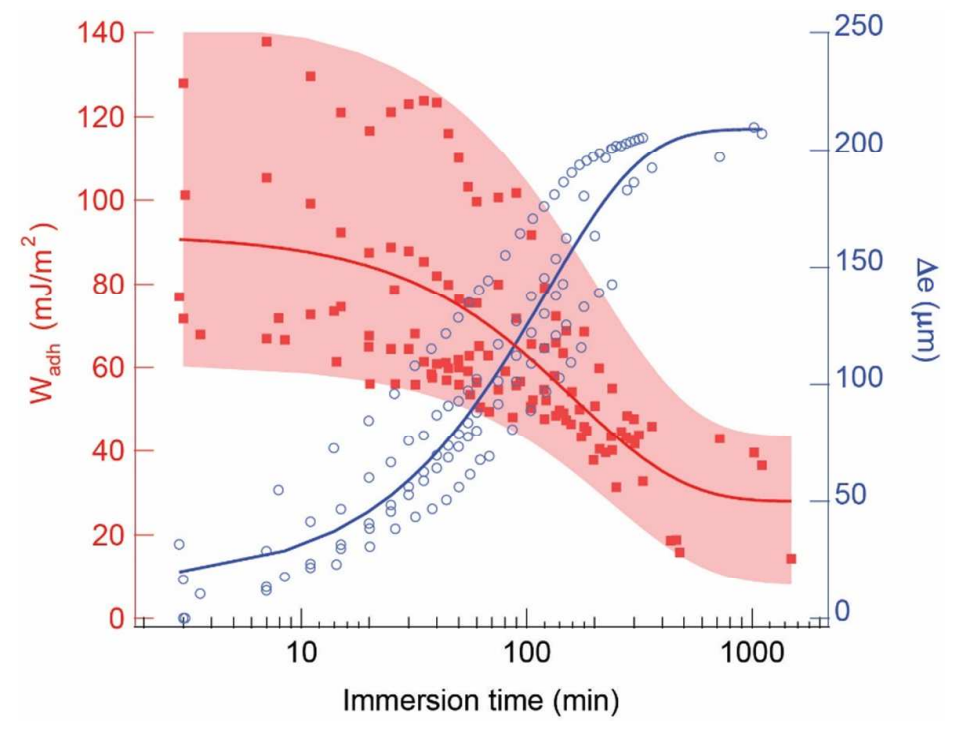

Figure 4: Kinetics of adherence and swelling of P10-CL2 hydrogels $(n=5)$ from preparation state to swelling equilibrium. On the left axis, the energy of adherence of the system measured as a function of immersion time is represented with the experimental data points $(\square)$, the dispersion ( $\square$ ) and the best fit of the data points with an arbitrary function to guide the eye $(-)\left(\mathrm{W}_{\mathrm{adh}}=\mathrm{A}_{0}+\mathrm{A}_{1} \mathrm{x} \exp \left(-\mathrm{t}_{\mathrm{imm}} / \tau_{1}\right)+\mathrm{A}_{2} \mathrm{x} \exp \left(-\mathrm{t}_{\mathrm{imm}} / \tau_{2}\right)\right.$ with $\mathrm{A}_{0}=27.9$ $\left.\mathrm{mJ} / \mathrm{m}^{2} ; \mathrm{A}_{1}=48.3 \mathrm{~mJ} / \mathrm{m}^{2} ; \mathrm{A}_{2}=15.5 \mathrm{~mJ} / \mathrm{m}^{2} ; \tau_{1}=20 \mathrm{~min}, \tau_{2}=90 \mathrm{~min}\right)$. On the right axis, the $i$ in situ variation of thickness of the sample is represented with the different experimental data points (o) and the fit of the data points $(-)\left(\Delta \mathrm{e}=\mathrm{B}_{0}+\mathrm{B}_{1} \mathrm{x} \exp \left(-\mathrm{t}_{\mathrm{imm}} / \tau_{3}\right)+\mathrm{B}_{2} \mathrm{x} \exp \left(-\mathrm{t}_{\mathrm{imm}} / \tau_{4}\right)\right.$ with $\mathrm{B}_{0}=209 \mu \mathrm{m} ; \mathrm{B}_{1}=-192 \mu \mathrm{m} ; \mathrm{B}_{2}=-2 \mu \mathrm{m} ; \tau_{3}=125$ $\left.\min , \tau_{4}=15 \mathrm{~min}\right)$. 
Simultaneously to the decrease in adherence, the thickness of the hydrogel, mechanically maintained with the Teflon coated plate, increased by approximatively $215 \mu \mathrm{m}$ corresponding to a swelling of $12 \%$ of the unconstrained part of the gel layer, where the contact is made with the polymer brush (Figure 4). This swelling ratio is very close to what is measured in one direction when the sample is free to swell in water (in unconstrained conditions Figure S2).

The progressive swelling of the hydrogel implies a dilution of the bulk concentration of polymer near the interface between the PDMA hydrogel and the PAA brush: in the case of the P10-CL2 hydrogel, this bulk dilution of the polymer is estimated at $29 \%$ from the preparation state to the swelling equilibrium, and should lead to a decrease of the probability of forming hydrogen bonds between the two surfaces.

\section{Evolution of the topography of the PDMA hydrogel}

During the in situ swelling, the mechanical holding of the hydrogel causes a slight macroscopic curvature of the top surface. The height difference caused by this curvature was estimated to approximately $10 \mu \mathrm{m}$ by comparing the indentation measurements of P10-CL2 in its preparation state and at swelling equilibrium (Fig. S3).

To complete our investigation of the evolution of the topography of the hydrogel once it is immersed in the aqueous solution and see if it can have an impact on the evolution of the adhesive properties of the gel, we also performed comparative AFM measurements in the liquid state on the P20-CL3 PDMA hydrogels (Figure 1). The difference in surface roughness between the gel in its preparation state (Figure 1A) and the gel at swelling equilibrium (Figure 1B) is of the order of a few nanometers. We also noticed the presence of holes, randomly distributed on the surface of swollen P20-CL3. The height of these holes is comprised between 12 and $25 \mathrm{~nm}$ and their average diameter is $350 \mathrm{~nm}$. The same structures have already been observed in the case of polymer thin films. The existence of these holes at swelling equilibrium is attributed to the partial dissolution of un-crosslinked polymer chains on the surface of the gel. The reorganization of the network by aggregation of hydrophilic units, leading to channels formation, helping the water transfer during the swelling, is another explanation discussed in the literature ${ }^{20-23}$.

The probe penetrates about $50 \mu \mathrm{m}$ deep in the gel during the contact phase. The roughness of a few nanometers and the curvature of $10 \mu \mathrm{m}$ of the swollen PDMA are completely flattened during the contact phase between the brush and the gel, regardless of its swelling state. The changes in topography observed on the PDMA hydrogel, due to its swelling and its mechanical holding during its immersion into aqueous medium, are unlikely to be responsible for the observed decrease of the energy of adherence. This assumption was confirmed by the comparison with the results of the same kinetic study performed on a P10-CL2 in its preparation state, maintained by chemical grafting on a glass substrate which does not involve any deformation of the gel (Figure S4-A). 


\section{$\underline{\text { Reversibility of the swelling }}$}

Because the gels in preparation conditions may contain unreacted molecules, we considered the possibility of a decrease in adherence due to a surface contamination or to the polymer degradation by hydrolysis. In order to check this hypothesis, we carefully and slowly dried a gel sample after equilibration to recover the initial degree of swelling in the preparation state. A new kinetic study of adherence during equilibration was then performed on this partially dried sample. The results of the adhesive test showed that after the drying step, the P10-CL2 hydrogel recovered the same level of adherence than it previously had in its preparation state (Figure S4-B). The evolution of the energy of adherence as a function of immersion time was identical during the second swelling test, and we observed the same decrease of adherence until a plateau value around $40 \mathrm{~mJ} \cdot \mathrm{m}^{-2}$.

\subsection{Effect of crosslinks density and polymer concentration on adherence}

To investigate the effect of the polymer concentration in the PDMA hydrogel on its adhesive properties with the PAA brush, kinetic studies of adherence and in situ swelling of PDMA hydrogels between preparation state and swelling equilibrium were carried out for a series of gels with a fixed monomer concentration (10 wt\%) and different crosslinks density (1, 2 and 3 mol\% relative to monomer) and for another series with a fixed crosslinking ratio ( $3 \mathrm{~mol} \%$ relative to monomer) and different initial concentrations of monomer (10, 15, 20 and $30 \mathrm{wt} \%)$. Figure 5 shows the in situ

swelling kinetics and the change in work of adherence as a function of immersion time for both cases as obtained from the same experimental protocol.

The kinetic studies of adherence performed on P10-CLy (5B) and Px-CL3 hydrogels in their preparation state (Figure 5A) confirmed the results already observed for P10-CL2. During the swelling process, the energy of adherence between the gel and the brush continuously decreases from an initial value corresponding to an out-of-equilibrium state close to the preparation state of the sample, to a minimal plateau value once the swelling equilibrium of the gel has been achieved. 


\section{A}
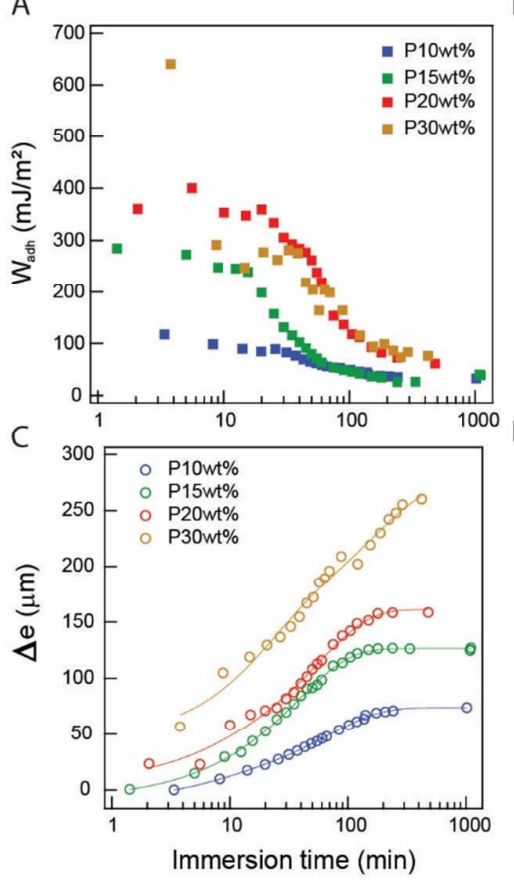

B
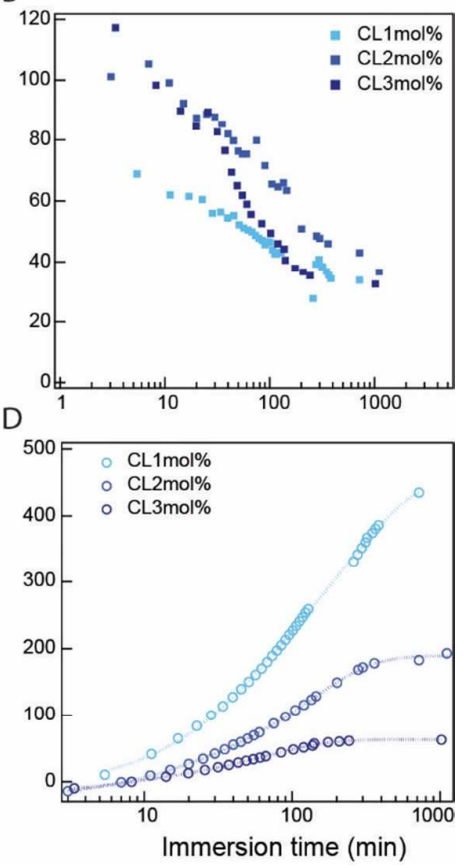

Figure 5 : Kinetics of adherence and swelling of Px-CLy hydrogels from preparation state to swelling equilibrium. (A) and (C) Energy of adherence $\mathrm{W}_{\text {adh }}$ and variation of the hydrogel thickness as a function of immersion time for different concentrations of polymer in the preparation state $(10,15,20$ and $30 \mathrm{wt} \%)$ and a constant crosslinking ratio fixed at $3 \mathrm{~mol} \%$. (B) and (D) Energy of adherence and variation of the hydrogel thickness as a function of immersion time for a constant initial concentration of polymer at preparation state of $10 \mathrm{wt} \%$ and different crosslinking ratios of 1,2 and $3 \mathrm{~mol} \%$.

At short (2-3 minutes) immersion times (Figure 5A), the energy of adherence of the PDMA hydrogel with the PAA brush increased more than 5 fold (from $117 \mathrm{~mJ} / \mathrm{m}^{2}$ to $640 \mathrm{~mJ} / \mathrm{m}^{2}$ ) with increasing initial concentration of monomer in the macroscopic gel (from 10 to $30 \mathrm{wt} \%$ ). In the same short immersion time conditions, a smaller increase of the energy of adherence was also observed (from 69 to 117 $\mathrm{mJ} / \mathrm{m}^{2}$ ) with increasing crosslinks density at fixed monomer concentration (Figure 5B). For long immersion times (equilibrium swelling) the same qualitative observations can be made concerning the influence of the concentration of polymer and of the crosslinks density but all values are much lower. $\mathrm{W}_{\text {adh }}$ at equilibrium swelling vary from $27 \mathrm{~mJ} / \mathrm{m}^{2}$ for P10-CL1 to $55 \mathrm{~mJ} / \mathrm{m}^{2}$ for P30-CL3 as summarized on Table 2. It is interesting to note that an equivalent level of adherence than P10-CL2 after 17 hours of contact (Figure 3), has been reach after 2 minutes of contact by the same gel but in its preparation state (Figure 5A).

\begin{tabular}{|l|c|c|c|c|c|c|c|}
\hline \multirow{2}{*}{$\begin{array}{c}\text { PDMA } \\
\text { P } x \text {-CLy }\end{array}$} & \multicolumn{6}{|c|}{ Out-of-equilibrium initial state $\left(\boldsymbol{t}_{\text {imm }}^{1}\right)$} & \multicolumn{4}{|c|}{ Swelling equilibrium state } \\
\cline { 2 - 8 } & $\begin{array}{c}W_{a d h}^{t_{i m m}^{1}} \\
\left(\mathrm{~mJ} . \mathrm{m}^{-2}\right)\end{array}$ & $\begin{array}{c}G_{0} \\
(\mathrm{kPa})\end{array}$ & $\begin{array}{c}C\left(t_{i m m}^{1}\right) \\
(\mathrm{wt} \%)\end{array}$ & $\begin{array}{c}\text { Swelling } \\
\text { equilibrium ratio } \\
\Lambda_{e q}\end{array}$ & $\begin{array}{c}W_{a d h}^{e q} \\
\left(\mathrm{~mJ} . \mathrm{m}^{-2}\right)\end{array}$ & $\begin{array}{c}G_{e q} \\
(\mathrm{kPa})\end{array}$ & $\begin{array}{c}C_{e q} \\
(\mathrm{wt} \%)\end{array}$ \\
\hline P10-CL1 & 69 & 13.5 & 8.8 & 16.5 & 27 & 11 & 6.0 \\
\hline P10-CL2 & 105 & 26 & 9.2 & 13.8 & 38 & 23 & 7.3 \\
\hline P10-CL3 & 117 & 36 & 9.8 & 10.9 & 33 & 37 & 9.1 \\
\hline P15-CL3 & 285 & 79 & 14.6 & 8.6 & 39 & 84 & 11.6 \\
\hline P20-CL3 & 400 & 127 & 18.7 & 6.8 & 48 & 122 & 14.4 \\
\hline P30-CL3 & 640 & 232 & 25.6 & 5.8 & 55 & 192 & 17.1 \\
\hline
\end{tabular}


Table 2: Adherence energy and gel composition at the bulk and near the interface in preparation and equilibrium conditions. $W_{a d h}^{t_{i m m}^{1}}$ refers to the energy of adherence measured at $t_{i m m}^{1} \approx 3 \mathrm{~min} . G_{0}$ is the shear modulus obtained by compression test at state preparation of the hydrogel and $G_{e q}$ at swelling equilibrium. $C\left(t_{i m m}^{1}\right)$ is the bulk concentration of polymer in the PDMA hydrogel at $t_{i m m}^{1} \approx 3 \min$ and $C_{e q}$ is the one at swelling equilibrium.

Given these results one may be tempted to attribute the decrease in adherence energy to the dilution of the polymer in the gel taking place during swelling and therefore to the decrease of the probability to form H-bond interactions between the gel and the PAA brush. It is therefore instructive to plot the adherence energy data, no longer as a function of swelling time but as a function of polymer concentration in the gel. Figure 6 shows the adherence energy as a function of polymer concentration for four different gels. Each color symbol corresponds to a different gel and experiments at all immersion times are compiled. It is immediately obvious from the data that the polymer concentration alone cannot explain the data.

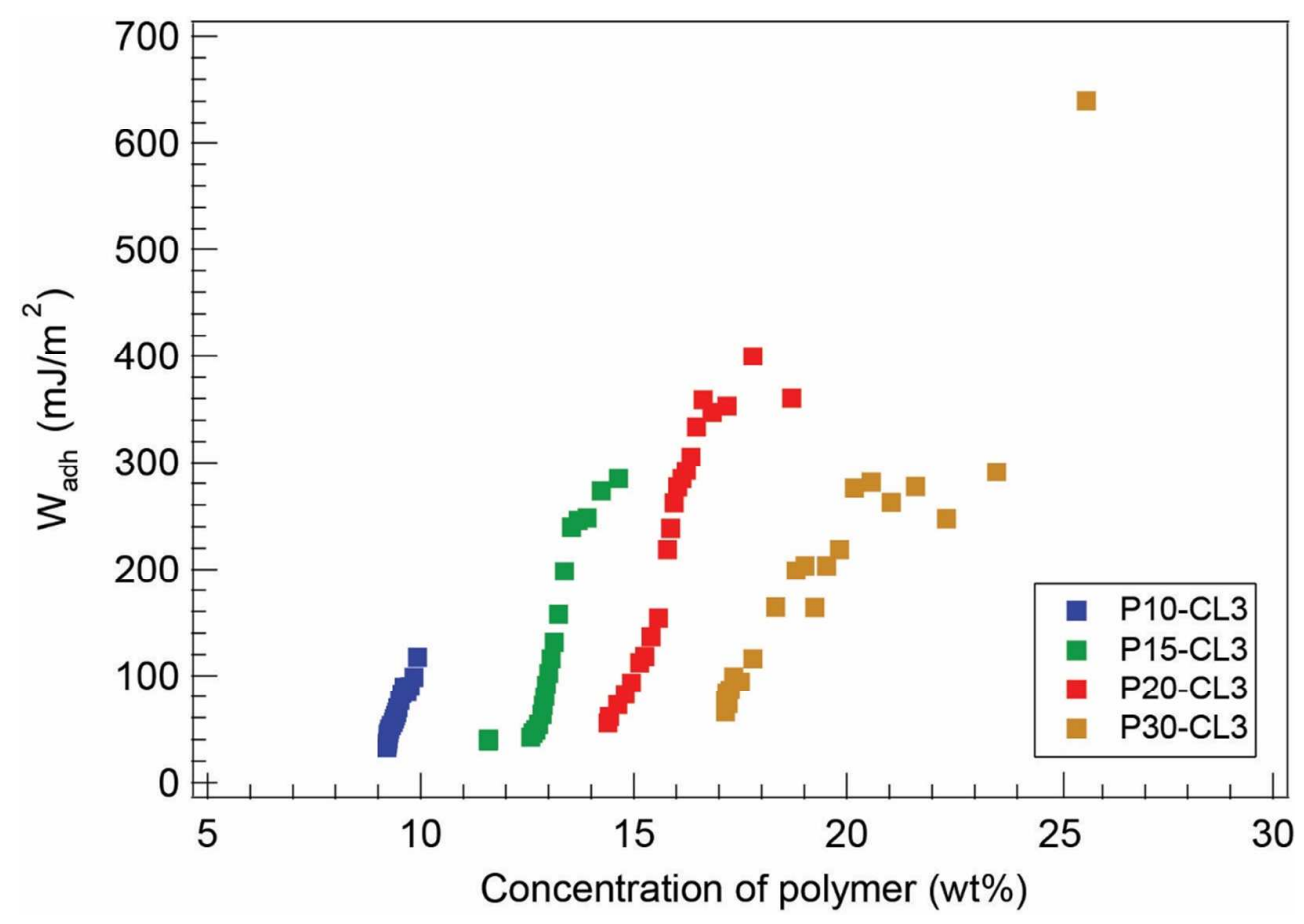

Figure 6 : Energy of adherence of $\mathbf{P} \boldsymbol{x}$-CL3 hydrogels as a function of the concentration of polymer in the hydrogel for $10,15,20$ and $30 \mathrm{wt} \%$.

A meaningful example is the comparison between P15-CL3 in its preparation state which has a polymer concentration around $14.4 \mathrm{wt} \%$ for $\mathrm{t}_{\mathrm{imm}}=1$ minute and P20-CL3 in its equilibrium state which has a polymer concentration of $14.6 \mathrm{wt} \%$ (Table 2 and Figure 6). The polymer concentration in the network is nearly identical but there is a large difference in adherence energy. The sample in its preparation state has an adherence energy $\mathrm{W}_{\mathrm{adh}}=285 \mathrm{~mJ} / \mathrm{m}^{2}$, while the sample at its swelling equilibrium has only $\mathrm{W}_{\mathrm{adh}}=48 \mathrm{~mJ} / \mathrm{m}^{2}$. 


\section{DISCUSSION}

Let us now try to determine what are the possible parameters influencing the measured adherence energy. An adherence test such as the one performed here can be divided in two stages. In the first stage, the interfacial interactions (hydrogen bonds in our case) between the two surfaces progressively form and we have seen that this process is rather slow at equilibrium (see Figure 3). In a second stage a stress is applied to the interface and the two surfaces are separated. Given the low value of adherence energy measured (well below $1 \mathrm{~J} / \mathrm{m}^{2}$ ) and, since our gels are quite elastic (Figure S5), one expects that the dissipated energy is more related to a molecular mechanism such as breakage of the multiple hydrogen bonds formed during the contact than to bulk viscoelastic dissipation. At the interface between the gel and the brush, hydrogen bonds keep forming and breaking based on kinetic mechanisms controlled by the association and dissociation constants of physical bonds. Given the strong dependence of the adherence energy on the contact time one can suppose that the concentration and strength (multiple bonds) of hydrogen bonds formed at the interfaces both increase with time.

For a given bond, the connection between the thermally activated bond constants and the bond scission when a force is applied has been made by Evans in his seminal series of paper on single bond scission as a function of loading rate ${ }^{24-25}$. In his picture, the activation energy for bond scission is modified by a factor $F \lambda$ when a force is applied. Chaudhury showed in a simplified model targeted at elastomer adhesion, that by applying this time-dependent bond scission to an extensible polymer chain, it is possible to account for the rate-dependent energy storage in the chain before bond breakage ${ }^{14-15}$. He considered a chain tethered permanently at one end and with a labile bond at the other end. When a force is applied, the chain stretches (it is an entropic spring), decreasing the energy barrier for breakage and consequently the probability of breakage. If the polymer chain is stretched at a constant speed $V$, the labile bond is also stretched at a constant speed $V_{\text {bond }}$ proportional to $V$. As the chain stretching rate increases the loading rate increases as well and the chain can be stretched further before breaking since the probability to break the bond is time and force dependent. In other words as $V$ increases, the time to break decreases but the force to break (and hence the stored elastic energy at the point of break) increases.

His model predicts for the adherence energy:

$$
W_{\text {adh }}=\left(\frac{\Sigma_{H B}}{2 k_{S}}\right)\left[\left(\frac{k T}{\lambda}\right) \ln \left(\frac{k_{s} V_{\text {bond }} \lambda \tau_{\text {diss }}}{k T}\right)\right]^{2} \quad \mathbf{E q . 3}
$$

where $\Sigma_{H B}$ is the areal density of hydrogen bonds formed at the interface, $\lambda$ is the activation length of the labile bond itself (a few $\mathrm{nm}$ ), $\tau_{\text {diss }}$ is the characteristic time of bond dissociation (depending on the bond strength), $k_{s}$ is the spring constant of the polymer chain and $V_{\text {bond }}$ is the pulling velocity on the bond. The term between square parentheses is the velocity dependent force at which the H-bonds break.

If one assumes that the pulling velocity on the bond is proportional to the macroscopic pulling velocity, this equation predicts that for a given interface and time of contact: 


$$
W_{a d h} \sim(\ln V)^{2} \quad \mathbf{E q . 4}
$$

which is in reasonable agreement with our experimental data for the adherence of P10-CL2 from a PAA brush in equilibrium conditions (Figure $7 \mathrm{~b}$ ). The slope of the linear fit of $\sqrt{W_{a d h}}$ as a function of $\ln V$ is equal to $0.032+/-0.003 \mathrm{~J}^{1 / 2} \mathrm{~m}^{-1}$ and the intercept point is equal to $0.46+/-0.15 \mathrm{~J}^{1 / 2} \mathrm{~m}^{-1}$ with a correlation coefficient $\mathrm{R}^{2}=0.984$ and the probe velocity in $\mathrm{m} / \mathrm{s}$. Hence experimentally, the faster the polymer chains are loaded, the higher is the force where the H-bond scission occurs and therefore the elastic energy dissipated during this bond scission process is higher.
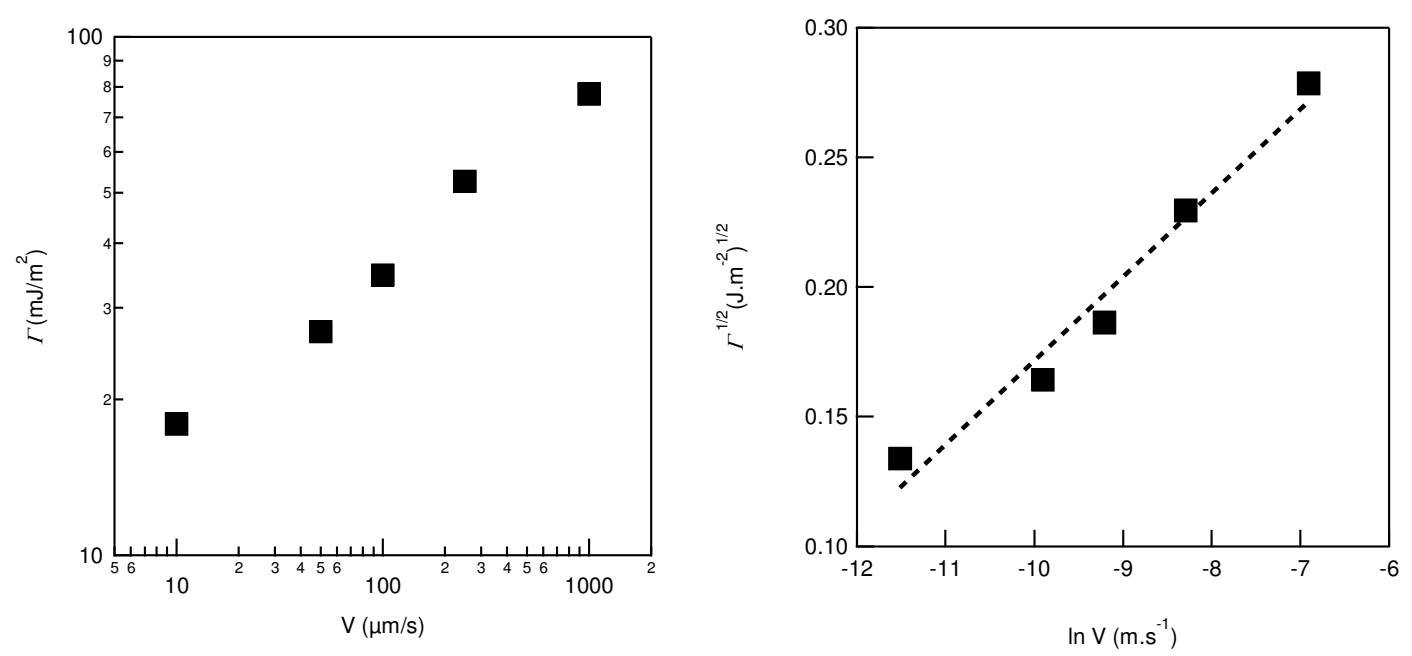

Figure 7 : Left: energy of adherence as a function of detachment speed of the polymer brush from the surface of P10-CL2 hydrogel at its swelling equilibrium. Right: Representation of the square root of the same data as a function of logarithm of detachment speed.

Using equation 3 and our fit of the square root of the energy of adherence as a function of logarithm of detachment speed, we can also estimate the order of magnitude of the dissociating constant of hydrogen bonds in our system. However we have three unknowns : $\Sigma_{H B}, k_{S}$ and $\tau_{\text {diss }}$ and only two equations. Following Chaudhury ${ }^{14}$ we assume that $\Sigma_{b}$ cannot be too different from the areal density of strands $\sim 10^{18}$ strands $/ \mathrm{m}^{2}$. This gives then a value of $k_{S}=0.8 \mathrm{~N} / \mathrm{m}$ close to the spring constant one would expect for a fully stretched chain $^{26}$ (see supplementary information for a more detailed discussion). This reflects the fact that to account for a measureable value of the adhesion energy the chain needs to be stretched before breaking and the bond strength cannot be too low. With this value of $k_{s}$ the dissociation time becomes $\tau_{\text {diss }}=8.7 \cdot 10^{-5} \mathrm{~s}$. We can then estimate with equation 5 the activation energy of dissociation of hydrogen bonds between the PDMA hydrogel and the PAA brush: 


$$
\tau_{\text {diss }}=\left(\frac{h}{k T}\right) \exp \left(\frac{E_{a}}{k T}\right) \mathbf{E q} .5
$$

which for P10-CL2 at its swelling equilibrium after a contact time of 120 seconds gives $E_{a}=20 \mathrm{kT}$ which is in a good agreement with what one would expect for multiple hydrogen bonds . It should be noted that for a different contact time the nature and areal density of formed bonds may be different and its activation energy might change.

In principle, equation 3 predicts also a Lake-Thomas like amplification factor of the adherence energy. In the prefactor $\left(\frac{\Sigma_{H B}}{2 k_{S}}\right), \Sigma_{H B}$ should scale with the concentration of polymer and increase with contact time while the spring constant of the polymer chain $k_{s}$ should scale with $N_{0}^{-1}$. This gives a prediction of $W_{a d h} \propto \Sigma_{H B}\left(t_{c}\right) N_{0}$, i.e. the adherence energy should scale with the inverse of the modulus of the gel ${ }^{17,27}$. However, for these weakly adhering systems the energy of the C-C covalent bond which is in the Lake and Thomas model, is replaced by a rate dependent strain energy which results from a balance between spring constant of the polymer strand and labile bond energy ${ }^{14}$.

Figure 8 shows that experimentally the adherence energy increases almost linearly with the modulus. Since the value of the spring constant $k_{S}$ in equation 3 has to scale with $1 / \mathrm{N}_{0}$, this suggests that the concentration and strength of multiple hydrogen bonds formed at the interface represented by $\Sigma_{H B}$ and $E_{a}$ must increase more than linearly with polymer concentration to compensate the spring constant effect.

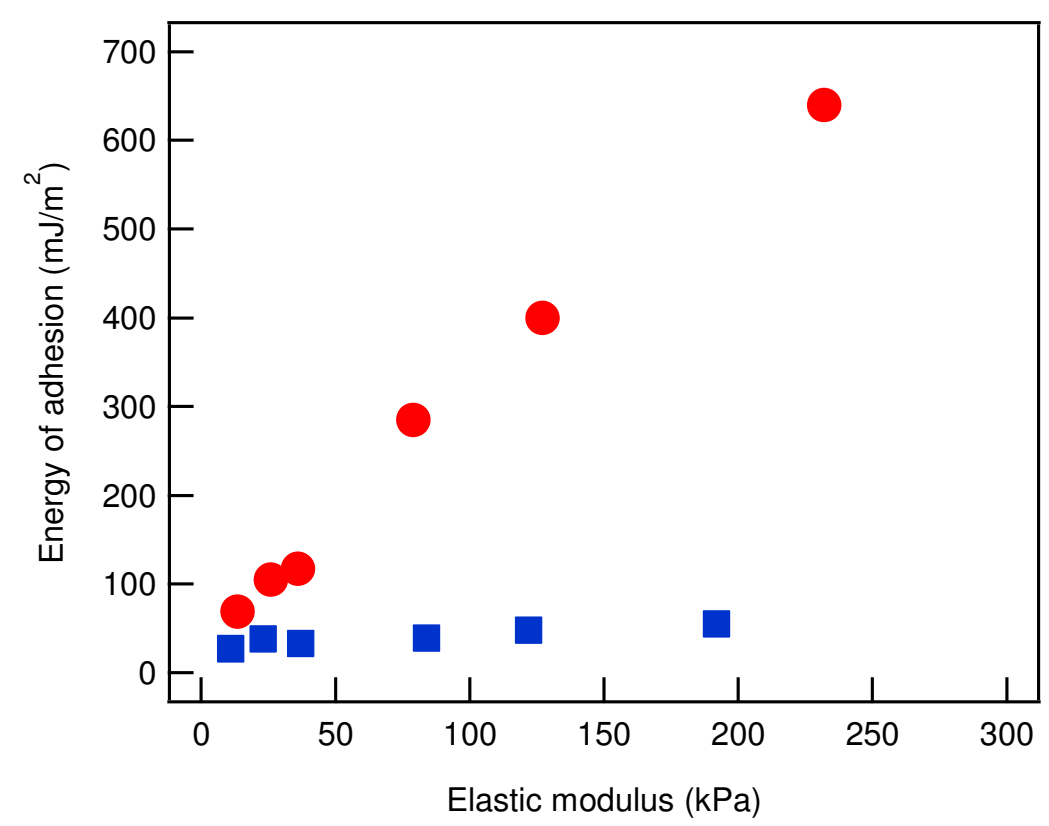

Figure 8 : Energy of adherence as a function of elastic modulus G of the PDMA hydrogel: red symbols are for hydrogels in their preparation state, and blue symbols for hydrogels swollen at equilibrium. 
In other words, for this system where adhesion is due to the formation of hydrogen bonds, the adherence energy increases with elastic modulus because more concentrated gels are able to form a higher concentration of stronger bonds faster.

This brings us now back to the question of why does $\Sigma_{H B}$ for a given contact time changes between the preparation conditions and the equilibrium conditions.

It has been $\operatorname{argued}^{28}$ that because the swelling stretches the polymer chains of the crosslinked network of the PDMA hydrogel, there is a decrease of degree of freedom for the polymer chains of the gel. This in turn would increase the entropic cost of forming multiple interactions. This thermodynamic argument is appealing but cannot explain how a very different adherence can be obtained with only a very small change in swelling ratio as for the P10-CL3 hydrogel.

An alternative argument relies on kinetics. The swelling rate of the PDMA gel, defined as the derivative of the swelling ratio $\Lambda(t)$ as a function of immersion time is:

$$
V_{\Lambda}(t)=\frac{d \Lambda}{d t} \quad \mathbf{E q . 6}
$$

If we plot the adherence energy as a function of $V_{\Lambda}$ (Figure S6), we are able to define for each composition a critical swelling flux, above which the gel is considered in an out-of-equilibrium state. We can also define a second regime when the swelling speed is close to zero, where the gel can be considered in its swelling equilibrium state.

Considering those two different regimes, we plotted the adherence energy (for the same contact time) as a function of the polymer concentration (Figure 9): for both conditions the adherence energy actually increases linearly with polymer concentration. However a significant difference exists in the magnitude of the effect. The slope is more than ten times larger in the case of the out-of-equilibrium state. If the expected dependence of the energy of adherence on the concentration of polymer is indeed observed, it cannot be the only factor controlling the level of adherence and the swelling state seems to play an important and significant part to the results of our study. 
One hypothesis that could be explored is linked to the non-fouling properties of hydrogels,

It is well-known that because of the hydration of the hydrophilic sites, the water shells formed along the polymer chains make the adherence of proteins through weak interactions impossible, acting like a repulsive barrier ${ }^{29}$. The expulsion of water molecules from both surface and protein is necessary to facilitate protein adsorption by reducing the free energy barrier arising from dehydration entropic effects.

In our case, all the chains are hydrophilic and shells of bound water are organized all around in both states. However, at $t_{i m m}^{1}$, the swelling is responsible of a water flux entering the gel. This flux could favor the destabilization of the water shells and increase the replacement and exchange of water molecules. We hypothesize that this flux could accelerate the reorganization of the H-bond interactions, in particular with the merging of hydration shells of hydrophilic sites and the formation of multiple intramolecular interactions between polymer chains, which are responsible of the adherence measured. Once the swelling equilibrium has been achieved, we hypothesize again that the exchange of bound water with free water slows down the probability to form multiple hydrogen between the polymer chains of the brush and the gel decreases. This scenario is drawn schematically in Figure 10. 

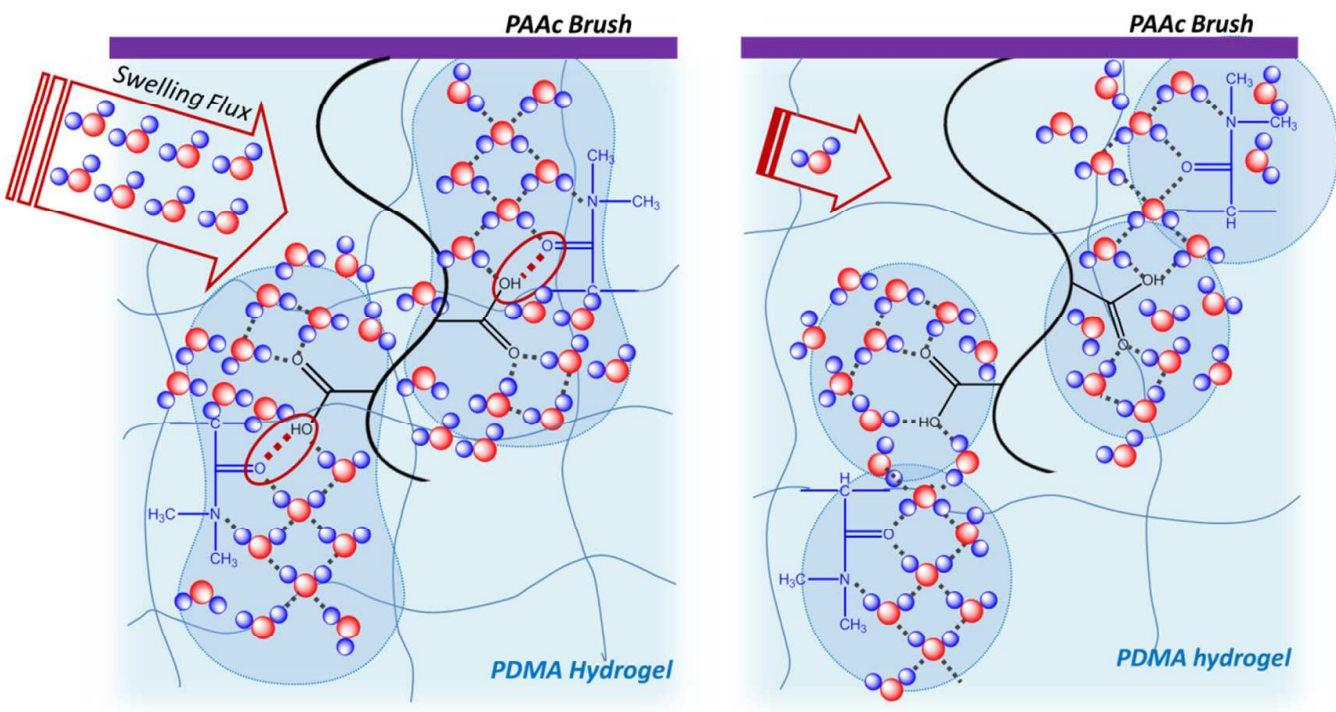

Figure 10: Schematic of the gel and brush with their water hydration shell. We speculate that the presence of a water flux may speed up the formation of hydrogen bonds.

If this hypothesis is correct one expects that the adherence that we measure has a different dependence on contact time if the contact is made in as-prepared or in equilibrated conditions. Therefore comparisons made at the same contact time may give indeed lower values of $\mathrm{W}_{\text {adh }}$ equilibrated gels than for dilute gels.

\section{CONCLUSION}

We have carried out systematic adherence experiments (at $\mathrm{pH}$ 2) between a neutral PDMA gel and a PAA brush by using a probe tack tester in immersed conditions. Our key finding is that regardless of the composition of the gels (monomer and cross-linker concentration), the measured value of adherence energy systematically decreases with increasing immersion time, i.e; as the gel swells to equilibrium. Interestingly for each specific condition (short or long immersion time) the adherence energy increases linearly with the elastic modulus of the gel i.e. with the bulk density of elastic chains which controls the adhesive interactions at the interface. When we analyze the debonding process however we find two interesting but contradictory results: on one hand for a given gel the adherence energy increases linearly with $(\ln V)^{2}$ suggesting that chain stretching occurs before failure, and on the other hand at a given debonding rate the adherence energy increases with the modulus suggesting that the dissipated energy does not depend on the length of the chain being stretched but rather on the density $\Sigma_{H B}$ of hydrogen bonds formed at the interface.

To reconcile these two results, we propose that the rate of formation of multiple hydrogen bonds at the interface and their activation energy (strength of the bond) may be a highly kinetic effect so that 
$\Sigma_{H B}(t)$ and $E_{a}(t)$ increase faster in the presence of a flux of water entering the gel during the swelling/deswelling process and with polymer concentration. If this is the case the lower adherence energy measured at equilibrium conditions (when the flux is low) may simply be due to the slower kinetics of multiple H-bond formation.

\section{REFERENCES}

1. Annabi, N.; Tamayol, A.; Shin, S. R.; Ghaemmaghami, A. M.; Peppas, N. A.; Khademhosseini, A., Surgical materials: Current challenges and nano-enabled solutions. Nano Today 2014, 9 (5), 574589.

2. Andrews, G. P.; Laverty, T. P.; Jones, D. S., Mucoadhesive polymeric platforms for controlled drug delivery. Eur J Pharm Biopharm 2009, 71 (3), 505-518.

3. Zhu, F.; Wang, C.; Yang, S.; Wang, Q.; Liang, F.; Liu, C.; Qiu, D.; Qu, X.; Hu, Z.; Yang, Z., Injectable tissue adhesive composite hydrogel with fibroblasts for treating skin defects. Journal of Materials Chemistry B 2017, 5 (13), 2416-2424.

4. Kang, H.-W.; Lee, S. J.; Ko, I. K.; Kengla, C.; Yoo, J. J.; Atala, A., A 3D bioprinting system to produce human-scale tissue constructs with structural integrity. Nat Biotechnol 2016, 34, 312.

5. Yuk, H.; Zhang, T.; Lin, S.; Parada, G. A.; Zhao, X., Tough bonding of hydrogels to diverse nonporous surfaces. Nat Mater 2016, 15 (2), 190-196.

6. Tang, J.; Li, J.; Vlassak, J. J.; Suo, Z., Adhesion between highly stretchable materials. Soft Matter 2016, 12 (4), 1093-1099.

7. Li, J.; Celiz, A. D.; Yang, J.; Yang, Q.; Wamala, I.; Whyte, W.; Seo, B. R.; Vasilyev, N. V.; Vlassak, J. J.; Suo, Z.; Mooney, D. J., Tough adhesives for diverse wet surfaces. Science 2017, 357 (6349), 378381.

8. Jiawei, Y.; Ruobing, B.; Zhigang, S., Topological Adhesion of Wet Materials. Adv Mater 2018, 0 (0), 1800671.

9. $\quad$ La Spina, R.; Tomlinson, M. R.; Ruiz-Perez, L.; Chiche, A.; Langridge, S.; Geoghegan, M., Controlling network-brush interactions to achieve switchable adhesion. Angew. Chem.-Int. Edit. 2007, 46 (34), 6460-6463.

10. Alfhaid, L.; Seddon, W. D.; Williams, N. H.; Geoghegan, M., Double-network hydrogels improve $\mathrm{pH}$-switchable adhesion. Soft Matter 2016, 12 (22), 5022-5028.

11. Sudre, G.; Olanier, L.; Tran, Y.; Hourdet, D.; Creton, C., Reversible adhesion between a hydrogel and a polymer brush. Soft Matter 2012, 8 (31), 8184 - 8193.

12. Lin, W. C.; Fan, W.; Marcellan, A.; Hourdet, D.; Creton, C., Large Strain and Fracture Properties of Poly (dimethyl acrylamide)/silica Hybrid Hydrogels. Macromolecules 2010, 43, 25542563.

13. Rose, S.; Dizeux, A.; Narita, T.; Hourdet, D.; Marcellan, A., Time Dependence of Dissipative and Recovery Processes in Nanohybrid Hydrogels. Macromolecules 2013, 46 (10), 4095-4104.

14. Chaudhury, M. K., Rate-Dependent Fracture at Adhesive Interface. The Journal of Physical Chemistry B 1999, 103 (31), 6562-6566.

15. Ghatak, A.; Vorvolakos, K.; She, H.; Malotky, D. L.; Chaudhury, M. K., Interfacial rate processes in adhesion and friction. Journal of physical chemistry B 2000, 104, 4018-4030.

16. Merkel, R.; Nassoy, P.; Leung, A.; Ritchie, K.; Evans, E., Energy landscapes of receptor-ligand bonds explored with dynamic force spectroscopy. Nature 1999, 397, 50-53.

17. Lake, G. J.; Thomas, A. G., The strength of highly elastic materials. Proceedings of the Royal Society of London, series A: Mathematical and Physical Sciences 1967, A300, 108-119.

18. Sudre, G.; Tran, Y.; Creton, C.; Hourdet, D., pH/Temperature control of interpolymer complexation between poly(acrylic acid) 
and weak polybases in aqueous solutions. Polymer 2012, 53, 379-385.

19. Sudre, G.; Siband, E.; Hourdet, D.; Creton, C.; Cousin, F.; Tran, Y., Synthesis and Characterization of Poly(acrylic acid) Brushes: "Grafting-Onto" Route. Macromolecular Chemistry and Physics 2012, 213, 293-300.

20. Tani, K.; Fujiyoshi, Y., Water channel structures analysed by electron crystallography. Biochimica et Biophysica Acta (BBA) - General Subjects 2014, 1840 (5), 1605-1613.

21. Kim, S. H.; Marmo, C.; Somorjai, G. A., Friction studies of hydrogel contact lenses using AFM: non-crosslinked polymers of low friction at the surface. Biomaterials 2001, 22 (24), 3285-3294.

22. Kim, S. H.; Opdahl, A.; Marmo, C.; Somorjai, G. A., AFM and SFG studies of pHEMA-based hydrogel contact lens surfaces in saline solution: adhesion, friction, and the presence of noncrosslinked polymer chains at the surface. Biomaterials 2002, 23 (7), 1657-1666.

23. Beines, P. W.; Klosterkamp, I.; Menges, B.; Jonas, U.; Knoll, W., Responsive Thin Hydrogel Layers from Photo-Cross-Linkable Poly(N-isopropylacrylamide) Terpolymers. Langmuir 2007, 23 (4), 2231-2238.

24. Evans, E.; Ritchie, K., Dynamic Strength of Molecular Adhesion Bonds. Biophys J 1997, 72, 1541-1555.

25. Evans, E. A.; Calderwood, D. A., Forces and bond dynamics in cell adhesion. Science 2007, 316 (5828), 1148-1153.

26. Hui, C. Y.; Tang, T.; Lin, Y. Y.; Chaudhury, M. K., Failure of elastomeric polymers due to rate dependent bond rupture. Langmuir 2004, 20 (14), 6052-6064.

27. Akagi, Y.; Sakurai, H.; Gong, J. P.; Chung, U.-i.; Sakai, T., Fracture energy of polymer gels with controlled network structures. The Journal of Chemical Physics 2013, 139 (14), 144905.

28. Rose, S.; Prevoteau, A.; Elziere, P.; Hourdet, D.; Marcellan, A.; Leibler, L., Nanoparticle solutions as adhesives for gels and biological tissues. Nature 2014, 505 (7483), 382-385.

29. Chen, S.; Li, L.; Zhao, C.; Zheng, J., Surface hydration: Principles and applications toward lowfouling/nonfouling biomaterials. Polymer 2010, 51 (23), 5283-5293. 